\title{
Tools for organizing training, part 4
}

\author{
Heather Waldis ${ }^{1}$, Christal Huber ${ }^{2} \&$ Bruce W. Kenned ${ }^{3}$
}

To conclude our series on tools for organizing training, we consider a computer tool for developing learning objectives. Recall that a learning objective is a statement that describes the specific skills or knowledge that a learner should be able to demonstrate after successful training. When designing learning objectives, verb choice is important, as certain terms can be too vague or fall short of providing a measurable outcome. A trainer must consider which actions can appropriately validate understanding. By writing learning objectives that contain quantifiable goals, trainers can better assess learning and how well trainees have met their objectives.

One of the many resources available to help trainers create constructive learning objectives is the Objectives Builder Tool (OBT), a free online application provided by Arizona State University (https://teachonline.asu.edu/objectives-builder). The OBT is based on Bloom's taxonomy and includes a tutorial to help trainers write instructional learning objectives while avoiding common pitfalls. Aided by the tool, trainers select an overview objective that corresponds to one of the cognitive levels of Bloom's taxonomy, review verb choices that correlate to the chosen level, and establish specific goals for learners to accomplish by the end of the training. In the OBT interface, the user first chooses the general type of learning objective that a trainee should be able to demonstrate after training. Once selected, the tool provides an overview describing the applicable level of Bloom's taxonomy. The OBT also provides a list of appropriate verbs for writing objectives at the chosen level as well as example objectives. As verbs are selected, they are loaded into the text editor, where the user can customize each learning objective with specific details. By adding various objectives at different cognitive levels, a user can create the framework for an entire lesson. In doing so, trainers create measurable objectives that allow training to be evaluated and documented. With the help of tools like the OBT and other systems of conceptualizing learning, trainers can develop organizational strategies for effective training and assessment of knowledge. We encourage you to explore these resources on your own.

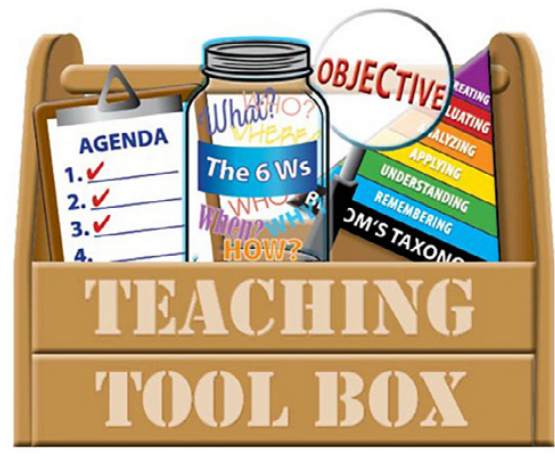

Training is a requirement in virtually all that we do, but it can be a challenge to train personnel efficiently and effectively, especially in research applications. However, by learning and practicing some of the tools that we have presented in this series, trainers can more easily organize their training. These tools can be used individually or synergistically to help frame and refine learning objectives, planned outcomes and goals for a training session. Ultimately, along with welfare for the animal, what is learned is what counts for the trainee.
${ }^{1}$ Charles River Laboratories, Frederick, MD.

${ }^{2}$ MFA Incorporated, Columbia, MO. ${ }^{3}$ California

State Polytechnic University, Pomona, CA.

Correspondence should be addressed to B.W.K.

(bkennedy@cpp.edu). 